\title{
LODOS RESIDUALES DE NEJAYOTE COMO SUSTRATOS PARA LA GERMINACIÓN DE SEMILLAS DE MAÍZ AZUL CRIOLLO
}

\author{
Victoria TÉLLEZ-PÉREZ1 ${ }^{1}$ Jesús F. LÓPEZ-OLGUÍN ${ }^{2}$ Agustín ARAGÓN² \\ y María Teresa ZAYAS-PÉREZ ${ }^{3 *}$
}

\footnotetext{
${ }^{1}$ Posgrado en Manejo Sostenible de Agroecosistemas, Instituto de Ciencias, Benemérita Universidad Autónoma de Puebla, Edificio VAL1, km 1.7 carretera a San Baltazar Tetela, 72960 Puebla, México

${ }^{2}$ Centro de Agroecología. Instituto de Ciencias, Benemérita Universidad Autónoma Puebla, ${ }^{3}$ Edificio VAL1, Km 1.7 Carretera a San Baltazar Tetela C.P. 72960, Puebla, México

${ }^{3}$ Centro de Química y Posgrado en Ciencias Ambientales, Instituto de Ciencias, Benemérita Universidad Autónoma de Puebla, Edificio IC8 Ciudad Universitaria, Col. San Manuel C.P. 72570 Puebla, México

*Author for correspondence: tzayasp@hotmail.com
}

(Recibido mayo 2017; aceptado noviembre 2017)

Palabras clave: nixtamalización, coagulación/floculación, Zea mays L., materia orgánica, agua residual, suelos arenosos

\section{RESUMEN}

Se evaluaron lodos del nejayote crudo (LNC) y lodos del nejayote tratado por coagulación/floculación (LNCF) como sustratos para la germinación de maíz azul proveniente de Amozoc de Mota, Puebla. Se estudiaron las características físicas y químicas de los LNC y LNCF para diferenciar sus propiedades. Se prepararon cuatro sustratos con distinto contenido de lodo y suelo: LNC25 y LNC50 con LNC; LNCF25 y LNCF50 con LNCF. El contenido de lodos en los sustratos fue de 25 y $50 \%$ en masa, respectivamente. Se usó suelo de la región (S) como referencia. El diseño experimental consistió en bloques al azar con cinco repeticiones de los cinco sustratos. La funcionalidad de los sustratos para la germinación se determinó con el porcentaje de germinación (PG) de 11 a 25 días después de la siembra (dds). Los datos se analizaron mediante varianza unifactorial y multifactorial. Los resultados muestran que a los 25 dds los PG del maíz fueron mayores o iguales que $62.5 \pm 9.4 \%$ en los diferentes sustratos. Con el sustrato LNC50 se obtuvieron PG $>90 \%$ de 15 a 25 dds, presentando diferencias significativas respecto a los demás. Por su bajo contenido de materia orgánica, los sustratos LNC25, LNCF25 y LNCF50 requirieron mayor tiempo para alcanzar los PG obtenidos con S. La concentración óptima de lodos y $\mathrm{S}$ junto con el contenido de materia orgánica posibilitan el aprovechamiento de lodos de nejayote para la germinación de maíz azul.

Key words: nixtamalization, coagulation/flocculation, Zea mays L., organic matter, wastewater, sandy soils

\begin{abstract}
Sludge obtained from raw nejayote (RNS) and sludge obtained from nejayote treated by coagulation/flocculation (SNCF) were evaluated as substrates for the germination of blue maize from Amozoc de Mota, Puebla. The RNS and SNCF were extracted by filtration and characterized in order to differentiate their physical and chemical properties. Four
\end{abstract}


substrates were prepared with different soil and sludge contents: RNS25 and RNS50 with RNS; SNCF25 and SNCF50 with SNCF. The sludge content in the substrates was 25 and $50 \%$ by mass, respectively. Soil of the region (S) was used as reference. The experiment was carried out under an experimental design of random blocks, with five replications of the five substrates. The functionality of the substrates for germination was determined with the germination percentage (GP) between 11 and 25 days after sowing (das). Data were analyzed with unifactorial and multifactorial ANOVA. The results show that at 25 das the GPs of maize were greater than or equal to $62.5 \pm 9.4 \%$ in the different substrates. GPs $>90 \%$ were obtained with the RNS50 substrate from 15 to 25 das, showing significant differences compared to the other substrates. Due to their low organic matter, the substrates RNS25, SNCF25 and SNCF50 required a longer time to reach the GP obtained with S. The optimum concentration of sludge and S, together with the organic matter content, enable the use of nejayote sludge for the germination of blue maize.

\section{INTRODUCCIÓN}

El tratamiento adecuado de los subproductos y residuos de las diferentes actividades industriales es una prioridad mundial. La problemática de los residuos en toda comunidad es compleja y su solución requiere un enfoque integral y sostenible (Zárate 2013). El aprovechamiento de los subproductos o residuos industriales ha sido un tema de suma importancia en las últimas décadas. Varios subproductos o residuos orgánicos de la industria agroalimentaria se han utilizado como sustratos para aumentar la materia orgánica del suelo y el rendimiento de los cultivos (Singh y Agrawal 2008, Papamichalaki et al. 2014, Sharma et al. 2017).

La principal función de los sustratos es proporcionar un soporte físico a las plantas para enraizarse y mantenerse erguidas, además de abastecerse de agua, oxígeno y nutrientes que mantengan el equilibrio del metabolismo y la fisiología vegetal (Díaz-Eliseche y Brassel 2008). Se considera que un sustrato ideal mantiene un $\mathrm{pH}$ y una estructura física estable, posee alta capacidad de aireación y retención de agua, está libre de arvenses y organismos patógenos, facilita el ahorro de agua y fertilizantes, es un producto rico en nutrientes esenciales, y su constitución es homogénea, renovable y biodegradable (Pinto et al. 2012, Nath 2014, Papamichalaki et al. 2014, Oliveira et al. 2015).

La germinación es considerada como la reanudación de la actividad metabólica en la semilla y es influida tanto por factores ambientales como por las propiedades físicas y químicas del sustrato (Oliveira et al. 2015). Distintos experimentos han demostrado que la composición del sustrato es el factor determinante de la germinación, supervivencia, crecimiento, fecundidad y mortalidad de las semillas. Conocer las propiedades físicas y químicas de un sustrato permite tomar decisiones acertadas sobre su manejo, con el fin de promover una germinación y crecimiento uniformes de semillas (Miralles et al. 2002, Gasparin et al. 2013, Oliveira et al. 2015). Adicionar al sustrato de germinación con materia orgánica a partir de aguas residuales puede incrementar la capacidad de retención de agua en suelos arenosos, mejorar la porosidad y aumentar tanto la biomasa como los nutrimentos disponibles (Nath 2014). Se ha reportado que los lodos de aguas residuales pueden aprovecharse en la agricultura (Ippolito et al. 2010) siempre que su manejo esté regulado por normas que establezcan los límites máximos permisibles de contaminantes (SEMARNAT 2002).

En México se conoce como nixtamalización al proceso de cocción del grano de maíz en presencia de hidróxido de calcio $\left(\mathrm{Ca}(\mathrm{OH})_{2}\right)$, y al agua residual de este procedimiento se le denomina "nejayote". La producción de nejayote es abundante, mayor a 50 millones de $\mathrm{m}^{3}$ /año (Scheel 2016) debido a la gran cantidad de nixtamal producido para la elaboración de tortilla de maíz, que es la base de la dieta alimenticia de la población mexicana (aproximadamente 119.5 millones de habitantes [INEGI 2016]).

El nejayote o agua residual del proceso de nixtamalización es de color amarillo y $\mathrm{pH} \geq 10$. Los sólidos sedimentables del nejayote son el endospermo y el pericarpio que se desprenden del grano de maíz durante la nixtamalización. Por tanto, en términos de composición, el nejayote es rico en materia orgánica, y está asociado con valores altos de la demanda química de oxígeno (DQO): 25 000-28 $000 \mathrm{mg} \mathrm{O}_{2} / \mathrm{L}$.

Generalmente, el nejayote o agua residual del proceso de nixtamalización se vierte en el drenaje municipal o en cuerpos de agua, suscitando un 
problema de contaminación ambiental. La separación de sólidos suspendidos, sedimentables y coloidales del nejayote se lleva cabo mediante operaciones de filtrado o el proceso físico y químico de coagulación/ floculación (Suárez et al. 2016, Téllez et al. 2016), La fase sólida o lodos del nejayote concentra la materia orgánica. El uso de coagulantes y floculantes naturales en el tratamiento del nejayote por coagulación/ floculación puede enriquecer el contenido de materia orgánica e iones metálicos en la fase sólida. Este proceso de coagulación/floculación como método de separación de sólidos coloidales es mucho más rápido y eficiente que el proceso de filtración. La composición química de los lodos que provienen del nejayote permite considerar su valor agrícola $\mathrm{y}$, consecuentemente, reducir el impacto ecológico negativo del nejayote en el ambiente.

El propósito de este trabajo fue evaluar el uso de lodos de nejayote crudo y lodos de nejayote tratado por coagulación/floculación como sustratos de germinación de maíz azul criollo (Z. mays) en invernadero, a fin de encontrar una alternativa al problema de contaminación por nejayote.

\section{MATERIALES Y MÉTODOS}

\section{Materiales utilizados en los ensayos}

El nejayote crudo usado en este trabajo se recolectó en un molino de nixtamal del municipio de Amozoc de Mota, Puebla, México. El nejayote, que es de color amarillo, denso y altamente alcalino, se almacenó en recipientes de plástico de $20 \mathrm{~L}$ y se refrigeró a $4{ }^{\circ} \mathrm{C}$ durante el trabajo experimental.

La separación de sólidos a partir del nejayote crudo se realizó por dos métodos: $a$ ) filtración y $b$ ) coagulación/floculación. El lodo proveniente del primer proceso es denominado lodo de nejayote crudo (LNC) en tanto que el derivado del segundo proceso es denominado lodo del nejayote tratado por coagulación/floculación (LNCF). Los sólidos obtenidos en ambos casos se secaron a temperatura ambiente. Los lodos LNC y LNCF, así como el suelo de la región fueron los materiales utilizados en la preparación de los sustratos para los ensayos de germinación de maíz azul.

\section{Caracterización física y química del nejayote crudo}

La caracterización física y química del nejayote se realizó con los métodos estándar de aguas residuales (APHA 2012). Los parámetros físicos y químicos de caracterización fueron los siguientes:
- pH y conductividad eléctrica medidos con un potenciómetro Conductronic PC 16.

- Demanda química de oxígeno (DQO), color y turbidez monitoreados con un fotómetro Merck 118. La DQO se evaluó por medio de viales (Merck, Alemania) con diferentes intervalos de medición. El método es análogo a EPA 410.4 (US Standard Methods 5220 D e ISO 15705).

- Sólidos totales (ST) y materia orgánica (MO) determinados con una mufla Thermolyn ${ }^{\circledR}$ Benchtop.

- Nitrógeno medido con un digestor de bloque Kjeldhal SEV®.

- Contenido de fósforo, potasio, calcio, magnesio, hierro, manganeso, cobre y zinc determinados con un espectrofotómetro de absorción atómica Perkin Elmer modelo AANALYST 200.

Separación de sólidos a partir del nejayote crudo

La separación de sólidos a partir del nejayote crudo se realizó por dos métodos: $a$ ) filtración y $b$ ) coagulación/floculación. El proceso de filtración de nejayote crudo se realizó a temperatura ambiente usando tela de algodón y una bomba de vacío. El proceso de coagulación/floculación del nejayote filtrado se realizó con los agentes Südflock ${ }^{\circledR}$ P-63 (aluminosilicato alcalino activado o bentonita alcalina) como coagulante y Sumex Biofloc ${ }^{\circledR}$ A-01 (poliacrilamida o polímero aniónico) como floculante.

El proceso de coagulación/floculación del nejayote fue estudiado previamente por Téllez et al. (2016), quienes encontraron las condiciones experimentales apropiadas de $\mathrm{pH}$, coagulante y floculante para obtener los valores más bajos de turbidez, color y DQO del nejayote crudo filtrado. Los resultados indicaron que a $\mathrm{pH}$ inicial de 9.0 se requiere una dosis de coagulante (Südflock ${ }^{\circledR} \mathrm{P}-63$ ) de 7.5 a $11.5 \mathrm{~g} / \mathrm{L}$ y una dosis de floculante (Sumex Biofloc ${ }^{\circledR}$ A-01) de $0.2 \times$ $10^{-3} \mathrm{~g} / \mathrm{L}$ para remover los sólidos sedimentables y coloidales del nejayote crudo. Los LNC y LNCF se secaron a temperatura ambiente y se utilizaron para preparar los sustratos con lodos de nejayote.

\section{Caracterización física y química del suelo y de los lodos residuales de nejayote}

La caracterización física y química de suelo y lodos (LNC y LNCF) se realizó con base en las Normas Oficiales Mexicanas NOM-021-SEMARNAT-2000 (SEMARNAT 2000) y NOM-147-SEMARNAT/ SSA1-2004 (SEMARNAT-SSA 2004).

Los parámetros físicos y químicos de caracterización del suelo y de lodos LNC y LNCF fueron los siguientes: densidad aparente en estado húmedo, humedad, sólidos totales, porosidad, retención de 
humedad, granulometría, peso, $\mathrm{pH}$, conductividad eléctrica, capacidad de intercambio catiónico (CIC), materia orgánica, nitrógeno total, fósforo, potasio, calcio, magnesio, hierro, manganeso y relación carbono/nitrógeno $(\mathrm{C} / \mathrm{N})$.

Los elementos químicos en la muestra en estado asimilable fueron identificados de acuerdo con su proceso de extracción en concordancia con la NOM021-SEMARNAT-2000 (SEMARNAT 2000) y cuantificados por espectrofotometría de absorción atómica.

\section{Preparación de los lodos de nejayote como sustra- tos para germinación}

Se determinó la cantidad de LNC y LNCF por litro de nejayote. Los lodos secos se molieron en un mortero hasta obtener gránulos menores o iguales a $20 \mathrm{~mm}$ de diámetro. En estas condiciones las partículas tienen la facilidad de incorporarse al suelo y aumentar la biodisponibilidad de los nutrimentos (Uicab-Brito y Sandoval-Castro 2003).

Los sustratos para germinación se prepararon mediante la mezcla de LNC o LNCF en distintas proporciones con suelo del terreno de siembra. El suelo utilizado es franco arenoso, proveniente del poblado de Amozoc de Mota, Puebla, México. El análisis físico y químico del suelo utilizado fue reportado por Téllez et al. (2016). La composición de los sustratos para la germinación en términos de la proporción de lodo y suelo se presenta en el cuadro I.

CUADRO I. SUSTRATOS ABASE DE LODOS DE NEJAYOTE PARA LA GERMINACIÓN DE SEMILLAS DE MAÍZ AZUL

\begin{tabular}{lcccc}
\hline Sustratos & $\begin{array}{c}\text { S } \\
(\%)\end{array}$ & $\begin{array}{c}\text { LNC } \\
(\%)\end{array}$ & $\begin{array}{c}\text { LNCF } \\
(\%)\end{array}$ & Símbolo \\
\hline $\begin{array}{l}\text { Control: suelo franco } \\
\text { arenoso de Amozoc de } \\
\text { Mota, Puebla, México }\end{array}$ & 100 & 0 & 0 & $\mathrm{~S}$ \\
\hline $\begin{array}{l}\text { Lodo de nejayote crudo al } \\
25 \%\end{array}$ & 75 & 25 & 0 & LNC25 \\
\hline $\begin{array}{l}\text { Lodo de nejayote crudo al } \\
50 \%\end{array}$ & 50 & 50 & 0 & LNC50 \\
\hline $\begin{array}{l}\text { Lodo de nejayote tratado } \\
\text { por coagulación/floculación } \\
\text { al } 50 \%\end{array}$ & 50 & 0 & 50 & LNCF50 \\
\hline $\begin{array}{l}\text { Lodo de nejayote tratado } \\
\text { por coagulación/floculación } \\
\text { al } 25 \%\end{array}$ & 75 & 0 & 25 & LNCF25 \\
\hline
\end{tabular}

S: suelo, LNC: lodo de nejayote crudo, LNCF: lodo de nejayote tratado por coagulación/floculación, \%: porcentaje de masa

\section{Aplicación de sustratos de lodos de nejayote para la germinación de maíz}

Los experimentos de germinación de semillas de maíz azul criollo (Zea mays L.) en los diferentes sustratos se realizaron en un invernadero de la Universidad Autónoma de Puebla, México, ubicado a $19^{\circ} 00^{\prime} 23.8^{\prime \prime}$ latitud norte, $98^{\circ} 12$ ' 23.0" latitud oeste y $2125 \mathrm{msnm}$. Las dimensiones del invernadero fueron de $7.5 \mathrm{~m}$ de largo, $4.5 \mathrm{~m}$ de ancho y $2.5 \mathrm{~m}$ de alto, con una cubierta de vidrio que mantuvo una temperatura interna promedio de $25^{\circ} \mathrm{C}$.

Los sustratos preparados se colocaron en charolas de polietileno y en cada cavidad se agregaron $0.1 \mathrm{~kg}$ de sustrato. El diseño experimental consistió en bloques al azar de cinco sustratos. Cada bloque correspondió a un sustrato con ocho unidades experimentales. Cada unidad experimental constó de una cavidad con dos semillas. Se realizaron cinco repeticiones por bloque con un total de 40 cavidades. Como indicador biológico se utilizaron semillas de maíz azul criollo (Z. mays L.) del grupo 1a cónico (Sánchez et al. 2000). Esta variedad se conoce como "maíz azul criollo" en la región de Amozoc de Mota, Puebla.

Para evaluar la funcionalidad de los sustratos para la germinación de maíz se determinó el PG para cada tratamiento entre los 11 y 25 días después de la siembra (dds).

\section{Análisis estadístico}

Los datos experimentales para cada sustrato se presentan por quintuplicado en términos de la media y el error estándar de los PG obtenidos. La evaluación estadística de la germinación de la semilla de maíz se realizó mediante análisis de varianza (ANDEVA) unifactorial, con la prueba de Bartlett y ANDEVA multifactorial con la prueba de Tukey $(\mathrm{p} \leq 0.05)$.

El análisis estadístico permite comparar las medias y determinar las diferencias significativas del efecto del sustrato. El análisis estadístico se realizó con el programa Statgraphics Centurion XVI v. 16.1.02.

\section{RESULTADOS Y DISCUSIÓN}

\section{Caracterización física y química del nejayote crudo}

Los parámetros físicos y químicos característicos de nejayote crudo se presentan en el cuadro II. El nejayote crudo es de color amarillo y posee valores elevados de $\mathrm{pH}$, turbidez, carga orgánica $(17146 \mathrm{mg}$ $\mathrm{O}_{2} / \mathrm{L}$ ) y conductividad eléctrica. El contenido de 
CUADRO II. PARÁMETROS FÍSICOS Y QUÍMICOS DEL NEJAYOTE CRUDO ${ }^{1}$

\begin{tabular}{lc}
\hline Parámetros & Nejayote crudo \\
\hline $\mathrm{pH}$ & 11.2 \\
Color $(1 / \mathrm{m})$ & 50.4 \\
Turbidez (NTU) & 072 \\
Sólidos totales (\%) & 1.08 \\
DQO (mg/L) & 17146 \\
Materia orgánica (\%) & 61.48 \\
Conductividad eléctrica (dS/m) & 3.95 \\
Nitrógeno total $(\%)$ & 0.08 \\
Fósforo (\%) & 0.015 \\
Potasio (\%) & 0.001 \\
Calcio (\%) & 0.941 \\
Magnesio (\%) & 0.174 \\
Hierro (\%) & 0.0011 \\
\hline
\end{tabular}

NTU: unidad nefelométrica de turbidez (por sus siglas en inglés), DQO: demanda química de oxígeno.

${ }^{1}$ Téllez et al. 2016

nitrógeno, fósforo, potasio, calcio, magnesio y hierro es inferior al $1 \%$. El contenido de calcio en nejayote es relativamente alto y proviene principalmente del hidróxido de calcio utilizado en el proceso de nixtamalización. La concentración de iones calcio en nejayote es relativamente elevada si se compara con efluentes de la industria láctea o de alimentos (Hung et al. 2006, Singh et al. 2012). El porcentaje de materia orgánica en nejayote es considerablemente alto y se atribuye a los residuos del pericarpio desprendido del grano de maíz, cuya composición, de acuerdo con Watson y Ramstad (1987), consiste en cenizas $(0.8 \%)$, almidón (7.3\%), azúcares (0.34 \%), proteínas (3.7\%), extracto etéreo ( $1 \%$ ) y fibra cruda $(86.7 \%)$.

\section{Caracterización física y química del suelo y de lodos de nejayote}

Los parámetros físicos y químicos de la caracterización del suelo y de los LNC y LNCF se presentan en el cuadro III. Una comparación de dichos parámetros muestra que con excepción del contenido de $\mathrm{Zn}$, el suelo presenta valores relativamente bajos de los demás parámetros (retención de humedad, $\mathrm{pH}$, capacidad de intercambio catiónico, materia orgánica, relación $\mathrm{C} / \mathrm{N}$, nitrógeno, potasio, calcio, hierro, manganeso), mientras que los lodos del nejayote muestran valores relativamente altos. Las cantidades de lodos de nejayote obtenidas por ambos métodos fueron de 0.029 y $0.013 \mathrm{~kg} / \mathrm{L}$, respectivamente.

Una comparación de los parámetros físicos y químicos de LNC y LNCF indica que la densidad, humedad, retención de humedad y distribución granulométrica presentan valores similares en ambas muestras. Sin embargo, la porosidad, el pH, la conductividad eléctrica, y el contenido de materia orgánica presentaron diferencias significativas.

La porosidad de LNCF es 12.8 veces mayor que la de LNC. El pH de LNCF se incrementa hasta 13.6 en relación con el $\mathrm{pH}$ de 10.5 de $\mathrm{LNC}$, es decir, el carácter básico es favorecido en lodos que provienen del proceso de coagulación/floculación. Ambos lodos se clasifican como "materiales alcalinos" de acuerdo con la NOM-021-SEMARNAT-2000 (SEMARNAT 2000). Con excepción de los iones calcio, los porcentajes de iones potasio, magnesio, hierro, manganeso $\mathrm{y}$ zinc en LNCF fueron menores que en LNC.

El contenido de materia orgánica en LNC es 4.3 veces mayor que en LNCF. Las diferencias fundamentales de las propiedades fisicoquímicas de LNC y LNCF se deben a que el tratamiento de nejayote para obtener LNCF implicó la adición de coagulante (Südflock ${ }^{\circledR}$ P-63) y floculante (Sumex Biofloc ${ }^{\circledR}$ A-01).

La alta porosidad de LNCF (Cuadro III) se explica por las características de la arcilla bentonita Südflock ${ }^{\circledR}$ P-63 usada como coagulante natural en el tratamiento del nejayote. Las arcillas poseen gran cantidad de microporos y su porcentaje de porosidad es de 50 a $70 \%$ (Monroe y Wicander 2014). La estructura porosa de la bentonita se compone prioritariamente de microporos con una cierta cantidad de mesoporos (Stanković et al. 2011).

Por el contario, la baja porosidad de LNC se atribuye a la estructura molecular de las sustancias que componen el nejayote y sus agregados. Durante la nixtamalización, los sitios porosos del pericarpio sufren cambios, en tanto que el almidón, los aglomerados de $\mathrm{CaCO}_{3}$, la fibra, etc., presentan diferentes formas y estructuras (Valderrama-Bravo et al. 2012). En consecuencia, el lodo residual de LNC, a partir del nejayote filtrado, mantiene espacios porosos conformados principalmente por mesoporos y macroporos. Además, Shanti et al. (2012) reportaron que la fibra de maíz produce un efecto aglutinante y generador de poros. Aunque la porosidad de LNC fue menor que la de LNCF, se sugiere que los tamaños de poro de los primeros promueven la aireación y penetración hídrica en un sustrato de germinación.

La conductividad eléctrica de LNCF $(6.23 \mathrm{dS} / \mathrm{m})$ fue considerablemente mayor que la de LNC $(1.11 \mathrm{dS} / \mathrm{m})$. De acuerdo con Abu-Hassanein et al. (1996), la conductividad eléctrica de un sustrato depende de diversos factores como densidad, contenido hídrico, contenido de arcilla y mineralogía de la arcilla. Arcillas como la bentonita tienen una alta conductividad 
CUADRO III. PARÁMETROS FÍSICOS Y QUÍMICOS DEL SUELO Y DE LODOS RESIDUALES DE NEJAYOTE: LODOS DE NEJAYOTE CRUDO (LNC) Y LODOS DE NEJAYOTE TRATADO POR COAGULACIÓN/FLOCULACIÓN (LNCF)

\begin{tabular}{|c|c|c|c|c|}
\hline \multicolumn{2}{|l|}{ Parámetros } & Suelo & $\mathrm{LNC}$ & $\mathrm{LNCF}$ \\
\hline \multicolumn{2}{|l|}{ Densidad aparente húmedo $(\mathrm{g} / \mathrm{mL})$} & 1.26 & 1.15 & 1.04 \\
\hline \multicolumn{2}{|l|}{ Humedad (\%) } & 12.2 & 81.89 & 84.69 \\
\hline \multicolumn{2}{|l|}{ Sólidos totales $(\%)$} & NA & 18.11 & 10.21 \\
\hline \multicolumn{2}{|l|}{ Porosidad (\%) } & 62.16 & 6.5 & 83 \\
\hline \multicolumn{2}{|l|}{ Retención de humedad (\%) } & 13.8 & 155 & 150 \\
\hline \multirow{5}{*}{ Granulometría (\% en peso) } & $>2 \mathrm{~mm}$ & ND & 9 & 8.5 \\
\hline & $>0.5 \mathrm{~mm}$ & ND & 38.5 & 33 \\
\hline & $>0.25 \mathrm{~mm}$ & ND & 22.5 & 19.5 \\
\hline & $>0.053 \mathrm{~mm}$ & ND & 19 & 22.5 \\
\hline & $<0.053 \mathrm{~mm}$ & ND & 11 & 16.5 \\
\hline \multicolumn{2}{|l|}{$\mathrm{pH}$} & 7.45 & 10.46 & 13.64 \\
\hline \multicolumn{2}{|l|}{ Conductividad eléctrica (dS/m) } & 1.42 & 1.11 & 6.23 \\
\hline \multicolumn{2}{|c|}{ Capacidad de intercambio catiónico $(\mathrm{cmol} / \mathrm{kg})$} & 1.40 & 5 & 2.5 \\
\hline \multicolumn{2}{|l|}{ Materia orgánica (\%) } & 0.90 & 78.23 & 18.03 \\
\hline \multicolumn{2}{|l|}{ Nitrógeno total (\%) } & 0.024 & 0.05 & 0.07 \\
\hline \multicolumn{2}{|l|}{ Potasio (\%) } & 0.004 & 0.01 & 0.003 \\
\hline \multicolumn{2}{|l|}{ Calcio $(\%)$} & 0.028 & 0.08 & 0.29 \\
\hline \multicolumn{2}{|l|}{ Magnesio (ppm) } & $<0.001$ & 100 & $<0.001$ \\
\hline \multicolumn{2}{|l|}{ Hierro (ppm) } & 0.0043 & 14.03 & 0.94 \\
\hline \multicolumn{2}{|l|}{ Manganeso (ppm) } & 0.0001 & 0.1 & 0.05 \\
\hline \multicolumn{2}{|l|}{ Zinc (ppm) } & 12.25 & 0.05 & 0.01 \\
\hline \multicolumn{2}{|l|}{ Boro (ppm) } & 0.75 & 5.18 & 5.76 \\
\hline \multicolumn{2}{|l|}{ Relación carbono/nitrógeno (C/N) } & 25.1 & 965.78 & 148.4 \\
\hline
\end{tabular}

NA: no aplica para análisis de suelo, ND: no determinado

*soluble en el extracto de saturación, **extraído con ácido del dietilen-triamino-pentaacético [DTPA], ***extraído con $\mathrm{CaCl}$, cuantificado con Azometina-H. En todos los casos la determinación se realizó por espectrofotometría de absorción atómica

superficial, por lo que la cantidad de bentonita en un lodo tendrá una fuerte influencia en su conductividad eléctrica.

No obstante, una conductividad eléctrica por arriba de $1.7 \mathrm{dS} / \mathrm{m}$ afecta negativamente la porosidad, densidad aparente y estabilidad estructural de un sustrato (Tejada y González 2005). Adicionalmente, el incremento en la conductividad de LNCF guarda relación con el incremento del pH (13.6) y el contenido de calcio.

El valor de la CIC en LNC $(5 \mathrm{cmol} / \mathrm{kg})$ excedió en $100 \%$ el valor en LNCF $(2.5 \mathrm{cmol} / \mathrm{kg})$. De acuerdo con Satje y Nelson (2009), el valor de la CIC en un suelo puede incrementarse al añadir bentonita. El incremento en el valor de la CIC se traduce en mayor capacidad para la retención de nutrientes. Este no fue 
el caso de LNCF, ya que la presencia de la bentonita no incrementó el valor de la CIC respecto al valor observado en LNC.

Comparativamente, los LNC presentan el valor más alto de CIC (que puede estar asociado con el alto contenido de materia orgánica [78.23\%]) respecto de los LNCF. De acuerdo con Williams (2010), la materia orgánica posee la propiedad de intercambiar cationes, de tal forma que incrementa la capacidad del sustrato para retener nutrientes $\left(\mathrm{NH}_{4}{ }^{+}, \mathrm{N}, \mathrm{K}^{+}, \mathrm{Ca}^{2+} \mathrm{y}\right.$ $\left.\mathrm{Mg}^{2+}\right)$. Algunos componentes de la materia orgánica actúan como quelatos, lo que facilita la disponibilidad de micronutrientes metálicos como $\mathrm{Zn}, \mathrm{Fe}, \mathrm{Cu}$ y Mn.

El análisis químico de LNC y LNCF no detectó la presencia de iones de $\mathrm{As}, \mathrm{Cd}, \mathrm{Cr}, \mathrm{Cu}, \mathrm{Pb}, \mathrm{Ni}$ y $\mathrm{Hg}$. En consecuencia, tanto LNC como LNCF se clasifican como materiales "excelentes" para su aprovechamiento de acuerdo con la SEMARNAT (2002).

Las concentraciones de nitrógeno total en ambos lodos es del mismo orden de magnitud, esto indica que el proceso de coagulación/floculación no influye en el contenido de nitrógeno total. Cabe destacar que las concentraciones reportadas de nitrógeno en el agua o en los lodos residuales de la nixtamalización, no han sido mayores a $0.08 \%$ (Téllez et al. 2016, Suárez et al. 2016, García-Depraect et al. 2017). No se detectó fósforo en ninguno de los lodos.

Adicionalmente, se ha señalado que la adición de compuestos con magnesio o calcio eleva el $\mathrm{pH}$ y genera el crecimiento radical (Cabrera 1999). El contenido de minerales en los sustratos es importante para proporcionar un ambiente químico favorable.

Los experimentos de campo han demostrado que la composición de los sustratos es un factor determinante en la germinación de semillas (Perrow y Davy, 2002). Yerima et al. (2015) indicaron que la germinación es la etapa más vulnerable en el desarrollo de un cultivo, y en ella desempeñan un papel determinante las características del sustrato; por tanto, conformar un sustrato a partir de suelo y materia orgánica mejora sus propiedades físicas, químicas y biológicas e incrementa su capacidad como medio de germinación.

\section{Germinación de maíz azul en sustratos a base de lodos de nejayote}

Los resultados del proceso de germinación de semillas de maíz azul en sustratos a base de lodos residuales de nejayote y suelo se muestran en el cuadro IV. Los resultados muestran los PG de las semillas de maíz azul en el intervalo de 11 a 25 dds.

En general, de acuerdo con el cuadro IV, a los 25 días de siembra los porcentajes de germinación del maíz azul fueron $\geq 62.5 \pm 9.4 \%$ en los diferentes sustratos (S, LNC25, LNC50, LNCF25 y LNCF50), lo cual demostró que más de la mitad de las semillas sembradas lograron germinar en los sustratos evaluados. De acuerdo con el ANDEVA ( $\mathrm{p} \leq 0.05)$, el PG en el sustrato LNC50 es el más alto de todos en el periodo de 11 a 25 dds y presenta una diferencia significativa total (c) de los 11 a los 17 dds y otra diferencia significativa total (b) de los 19 a los 25 dds, respecto al efecto producido por los demás sustratos. Cabe resaltar que a partir del día 15 después de la siembra, el PG fue mayor al $90 \%$. Los PG obtenidos con el sustrato de referencia (S) mostraron una tendencia similar al obtenido en el sustrato LNC50. Sin embargo, los porcentajes de germinación fueron inferiores en todo el intervalo de tiempo (menor o igual al $75 \pm 5.5 \%$ ). En este caso se presentan tres grupos de medias (c, bc y ab) que describen diferencias significativas en el PG durante el periodo de 11 a 25 dds. Por otra parte, los PG de maíz azul en los sustratos LNC25, LNCF25 y LNCF50 muestran una tendencia similar con el tiempo: inicialmente (11 dds), los PG son inferiores al de referencia (S); posteriormente, los PG se incrementan y tienden al

CUADRO IV. PORCENTAJE DE GERMINACIÓN DE SEMILLAS DE MAÍZ DE LOS 11 A LOS 25 DÍAS DESPUÉS DE LA SIEMBRA

\begin{tabular}{lcccccccc}
\hline \multirow{8}{*}{ SE } & \multicolumn{7}{c}{ Porcentaje de germinación (\%) } \\
\cline { 2 - 9 } & \multicolumn{1}{c}{11 días } & 13 días & 15 días & 17 días & 19 días & 21 días & 23 días & 25 días \\
\hline $\mathrm{S}$ & $61.3 \pm 7.5^{\mathrm{c}}$ & $66.3 \pm 6.4^{\mathrm{bc}}$ & $68.8 \pm 7.1^{\mathrm{bc}}$ & $72.5 \pm 7.0^{\mathrm{bc}}$ & $72.5 \pm 7.0^{\mathrm{ab}}$ & $73.8 \pm 6.0^{\mathrm{ab}}$ & $73.8 \pm 6.0^{\mathrm{ab}}$ & $75.0 \pm 5.5^{\mathrm{ab}}$ \\
LNC25 & $2.50 \pm 2.5^{\mathrm{a}}$ & $8.75 \pm 8.7^{\mathrm{a}}$ & $30.0 \pm 6.3^{\mathrm{a}}$ & $37.5 \pm 6.8^{\mathrm{a}}$ & $55.0 \pm 5.0^{\mathrm{a}}$ & $62.5 \pm 5.9^{\mathrm{a}}$ & $71.3 \pm 6.7^{\mathrm{ab}}$ & $72.5 \pm 7.2^{\mathrm{ab}}$ \\
LNC50 & $66.3 \pm 3.7^{\mathrm{c}}$ & $86.3 \pm 3.6^{\mathrm{c}}$ & $91.3 \pm 1.5^{\mathrm{c}}$ & $92.5 \pm 2.3^{\mathrm{c}}$ & $93.8 \pm 1.9^{\mathrm{b}}$ & $93.8 \pm 1.9^{\mathrm{b}}$ & $93.8 \pm 1.9^{\mathrm{b}}$ & $93.8 \pm 1.9^{\mathrm{b}}$ \\
LNCF25 & $30.0 \pm 6.9^{\mathrm{b}}$ & $38.8 \pm 6.3^{\mathrm{b}}$ & $41.3 \pm 6.7^{\mathrm{a}}$ & $48.8 \pm 8.9^{\mathrm{ab}}$ & $57.5 \pm 9.5^{\mathrm{a}}$ & $58.8 \pm 9.4^{\mathrm{a}}$ & $62.5 \pm 9.4^{\mathrm{a}}$ & $62.5 \pm 9.4^{\mathrm{a}}$ \\
LNCF50 & $28.8 \pm 7.5^{\mathrm{b}}$ & $38.8 \pm 6.0^{\mathrm{b}}$ & $51.3 \pm 6.3^{\mathrm{ab}}$ & $60.0 \pm 5.8^{\mathrm{ab}}$ & $66.3 \pm 5.0^{\mathrm{a}}$ & $68.8 \pm 5.2^{\mathrm{ab}}$ & $77.5 \pm 4.6^{\mathrm{ab}}$ & $82.5 \pm 5.0^{\mathrm{ab}}$ \\
\hline
\end{tabular}

SE: sustratos evaluados, S: suelo, LNC: lodo de nejayote crudo, LNCF: lodo de nejayote tratado por coagulación/floculación Medias identificadas con la misma letra en el sentido de las columnas significan que no existe diferencia estadísticamente significativa entre ellas $(\mathrm{p} \leq 0.05)$ 
valor máximo obtenido con el sustrato de referencia $(75.0 \pm 5.5 \%)$. De esta forma, a lo largo del periodo (11-25 dds) los PG en el sustrato LNC25 presentan dos grupos de medias (a y ab), siendo (a) el grupo de medias más bajo de todos los sustratos. En los sustratos LNCF25 y LNCF50, los porcentajes de germinación evolucionaron similarmente y presentan tres grupos de medias $(a, b, a b)$ que establecen las diferencias estadísticamente significativas con la influencia del sustrato. Finalmente, al 25 dds los PG en LNC25 (72.5 $\pm 7.2 \%)$ y en LNCF25 (62.5 \pm $9.4 \%$ ) son muy cercanos al valor de referencia $(\mathrm{S})$, mientras que el PG en el sustrato LNCF50 es el más alto de los tres con una media de $(82.5 \pm 5.0 \%)$, pero inferior al de LNC50 (93.8 $\pm 1.9 \%)$. Resulta interesante observar que los sustratos LNC25, LNCF25 y LNCF50, con bajo contenido de materia orgánica, requieren de tiempos prolongados para alcanzar los valores máximos del sustrato de referencia, mientras que el LNC50 con alto contenido de materia orgánica a partir de lodos de nejayote crudo, alcanza altos porcentajes de germinación de semillas de maíz azul desde la etapa inicial (11 dds). Por tanto, el contenido óptimo de materia orgánica en el sustrato es sustancial para favorecer el proceso de germinación de la semilla de maíz azul.

Los valores altos de CIC y porcentaje de materia orgánica asociados con el sustrato LNC50 podrían considerarse factores que favorecen la disponibilidad de nutrimentos catiónicos disponibles y el mejoramiento de la estructura del sustrato, lo cual posibilitó una germinación favorable de la semilla de maíz. Con base en el análisis estadístico de los PG, se determinó que el incremento en la proporción de 25 a $50 \%$ de LNC en el sustrato aumentó de forma considerable el PG de semillas de maíz. De esta manera el sustrato, el LNC50 logró retener agua, otorgó apoyo físico a la planta y permitió un adecuado intercambio gaseoso entre el sistema radicular y la atmósfera.

De acuerdo con los PG obtenidos, los LNC y LNCF funcionaron como componentes de sustratos de germinación de semillas de maíz azul. Sin embargo, dadas las características físicas y químicas de LNC y LNCF, es recomendable utilizarlos como enmienda en sustratos con bajos niveles de $\mathrm{pH}$. Adicionalmente, los LNCF, cuya conductividad eléctrica es de $6.23 \mathrm{dS} / \mathrm{m}$, pueden utilizarse en sustratos con baja conductividad. Una conductividad eléctrica de 2 a $3.5 \mathrm{dS} / \mathrm{m}$ se considera apropiada para la mayoría de los cultivos, de 3.5 a 5 se considera ligeramente alta (para plantas resistentes a la salinidad) y de 0.75 a 1.99 se considera baja, adecuada para semilleros y plántulas (Delgado et al. 2016). Por lo anterior, se asume que las principales propiedades de los lodos que beneficiaron la germinación de semillas de maíz en los sustratos evaluados fueron el contenido de materia orgánica, la concentración de calcio, la CIC y la porosidad, de modo que el uso de LNC y LNCF en sustratos de germinación es una opción para el manejo de estos residuos.

\section{CONCLUSIONES}

Con base en sus características físicas y químicas, existen diferencias importantes en la composición y propiedades de los LNC y LNCF. En cada uno de los sustratos elaborados, el contenido porcentual de lodos fue de 25 o $50 \%$ en masa. Después de 25 dds, los porcentajes de germinación de maíz azul fueron $\geq 62.5 \pm 9.4 \%$ en los diferentes sustratos (S, LNC25, LNC50, LNCF25 y LNCF50), lo cual demostró que más de la mitad de las semillas sembradas lograron germinar en los sustratos evaluados. El sustrato LNC50 fue el que favoreció en el menor tiempo (11 a 25 dds) la germinación. Los sustratos LNC25, LNCF25 y LNCF50, con bajo contenido de materia orgánica, requieren de tiempos prolongados para que se presente la germinación de las semillas, al igual que en el sustrato de referencia (S). Por tanto, el contenido óptimo de materia orgánica en el sustrato a partir de LNC es sustancial para favorecer el proceso de germinación de semillas de maíz azul. Los lodos residuales del proceso de la nixtamalización son sustratos viables para la germinación de dichas semillas.

\section{AGRADECIMIENTOS}

Los autores agradecen el apoyo financiero brindado por el proyecto de la Vicerrectoría de Investigación y Estudios de Posgrado 2017, BUAP. Victoria Téllez también agradece la beca otorgada por el Consejo Nacional de Ciencia y Tecnología.

\section{REFERENCIAS}

Abu-Hassanein Z., Benson C., Wang X. y Blotz L. (1996). Determining bentonite content in soil-bentonite mixtures using electrical conductivity. Geotech. Test. J. 19 (1), 51-57. DOI: 10.1520/GTJ11407J

APHA (2012). Standard methods for examination of water and wastewater. 22a ed. American Public Health Association. Washington, EUA, 1496 pp. 
Cabrera R.I. (1999). Propiedades, uso y manejo de sustratos de cultivo para la producción de plantas en maceta. Rev. Chapingo Ser. Hortic. 5 (1), 5-11.

Delgado M.M., Miralles de Imperial R., Masaguer A. y Martín J.V. (2016). Estudio de turbas y residuos avícolas procedentes de pollo de engorde como componente de sustratos de cultivo. Rev. Int. Contam. Ambie. 32 (4), 455-462.

DOI: 10.20937/RICA.2016.32.04.09

Díaz-Eliseche C. y Brassel R.O. (2008). Diferentes proporciones de mezcla de un sustrato comercial con un sustrato casero sobre la germinación de tomate ( $\mathrm{Lyco}$ persicum sculentum Mill) hibrido «KOMBAT» y maíz (Zea mays) tipo chipa. Investig. Agrar. 10 (1), 65-69.

García-Depraect O., Gómez-Romero J., León-Becerril E. y López-López A. (2017). A novel biohydrogen production process: Co-digestion of vinasse and nejayote as complex raw substrates using a robust inoculum. Int. J. Hydrog. Energy 42 (9), 5820-5831. DOI: 10.1016/j.ijhydene.2016.11.204

Gasparin E., Araujo M.M., Tolfo C.V., Foltz D.R.B. y Magistrali P.R. (2013). Substrates for germination and physiological quality of storage seeds of Parapiptadenia rigida (Benth.) Brenan. J. Seed Sci. 35 (1), 77-85. DOI: $10.1590 / \mathrm{S} 2317-15372013000100011$

Hung Y.T., Britz T.J. y van Schalkwyk C. (2006). Treatment of dairy processing waste- waters. En: Waste treatment in the food processing industry (Wang L.K., Hung Y.T., Lo H.H. y Yapijakis C., Eds.). CRC Press Taylor and Francis, Boca Raton, Florida, EUA, pp. $1-28$.

INEGI (2016). Tabulados de la encuesta intercensal 2015. Población total de los Estados Unidos Mexicanos. Instituto Nacional de Estadística y Geografía. [en línea] http://www.beta.inegi.org.mx/proyectos/enchogares/ especiales/intercensal/ 11/11/2017.

Ippolito J.A., Barbarick K.A., Paschke M.W. y Brobst R.B. (2010). Infrequent composted biosolids applications affect semi-arid grassland soils and vegetation. J. Environ. Manage. 91 (5), 1123-1130.

DOI: 10.1016/j.jenvman.2010.01.004

Miralles de Imperial R., Beltrán E.M., Porcel M.A., Delgado M.M., Beringola M.L., Martín J.V., Calvo R. y Walter I. (2002). Emergencia de seis cultivos tratados con lodo, fresco y compostado, de estaciones depuradoras. Rev. Int. Contam. Ambie. 18 (3), 139-146.

Monroe J.S. y Wicander R. (2014). The changing earth: Exploring geology and evolution. 7a. ed. Cengage Learning, Stamford, Connecticut, EUA, 736 pp.

Nath T.N. (2014). Soil texture and total organic matter content and its influences on soil water holding capacity of some selected tea growing soils in Sivasagar, District of Assam, India. Int. J. Chem. Sci. 12 (4), 1419-1429.
Oliveira A.K.M., Alves S., Santos J. y Braga J.M. (2015). Temperature and substrate influences on seed germination and seedling formation in Callisthene fasciculata Mart. (Vochysiaceae) in the laboratory. Rev. Árvore 39 (3), 487-495.

DOI: 10.1590/0100-67622015000300009

Papamichalaki M., Papadaki A. y Tzortzakis N. (2014). Substitution of peat with municipal solid waste compost in watermelon seedling production combined with fertigation. Chilean J. Agric. Res. 74 (4), 452-459. DOI: $10.4067 / \mathrm{S} 0718-58392014000400012$

Perrow M.P. y Davy A.J. (2002). Handbook of ecological restoration, vol. 2. Cambridge University Press, Nueva York, EUA, 432 pp.

Pinto A., Freitas G., Gonçalves N., Flávio de Freitas Ramos H. y Silva I. (2012). Test germination of corn seeds in different environments. Applied Research and Agrotechnology 5 (3), 17-26.

DOI: $10.5777 /$ paet.v5i3.1900

Sánchez J.J., Goodman M.M. y Stuber C.W. (2000). Isozymatic and morphological diversity in the races of maize of Mexico. Econ. Bot. 54 (1), 43-59. DOI: 10.1007/BF02866599

Satje A. y Nelson P. (2009). Bentonite treatments can improve the nutrient and water holding capacity of sugarcane soils in the wet tropics. Proc. Aust. Soc. Sugar Cane Technol. 31, 166-176.

Scheel C. (2016). Beyond sustainability. Transforming industrial zero-valued residues into increasing economic returns. J. Clean Prod. 131, 376-386. DOI: 10.1016/j.jclepro.2016.05.018.

SEMARNAT (2000). Norma Oficial Mexicana NOM021-RECNAT-2000. Que establece las especificaciones de fertilidad, salinidad y clasificación de suelos, estudio, muestreo y análisis. Secretaria de Medio Ambiente y Recursos Naturales. Diario Oficial de la Federación, 31 de diciembre de 2002.

SEMARNAT (2002). Norma Oficial Mexicana NOM004-SEMARNAT-2002. Protección ambiental.- Lodos y biosólidos.- Especificaciones y límites máximos permisibles de contaminantes para su aprovechamiento y disposición final. Secretaria de Medio Ambiente y Recursos Naturales. Diario Oficial de la Federación, 15 de agosto de 2003.

SEMARNAT-SSA (2004). Norma Oficial Mexicana NOM-147-SEMARNAT/SSA1-2004. Que establece criterios para determinar las concentraciones de remediación de suelos contaminados por arsénico, bario, berilio, cadmio, cromo hexavalente, mercurio, níquel, plata, plomo, selenio, talio y/o vanadio. Secretaría de Medio Ambiente y Recursos Naturales - Secretaría de Salud. Diario Oficial de la Federación, 2 de marzo de 2007. 
Shanti N.O., Bierschenk D.M., Barnett S.A. y Faber K.T. (2012). Direct lamination of solid oxide fuel cell anode support, anode, and electrolyte by sequential tape casting of thermoreversible gel slips. J. Power Sources 212 (1), 43-46. DOI: 10.1016/j.jpowsour.2012.03.073

Sharma B., Sarkar A., Singh P. y Pratap R. (2017). Agricultural utilization of biosolids: A review on potential effects on soil and plant grown. Waste Manag. 64, 117-132. DOI: 10.1016/j.wasman.2017.03.002

Singh R.P. y Agrawal M. (2008). Potential benefits and risks of land application of sewage sludge. Waste Manag. 28 (2), 347-358.

DOI: 10.1016/j.wasman.2006.12.010

Singh S.N., Srivastava G. y Bhatt A. (2012). Physicochemical determination of pollutants in wastewater in Dheradun. Curr. World Environ. 7 (1), 133-138.

DOI: 10.12944/CWE.7.1.20

Stanković N., Logar M., Luković J., Pantić J., Miljević M., Babić B. y Radosavljević-Mihajlović A. (2011). Characterization of bentonite clay from "Greda" deposit. Process. Appl. Ceram. 5 (2), 97-101. DOI: $10.2298 /$ pac1102097s

Suárez K.A., Ponce S.M., López J.T., Cornejo J.M., Oropeza M.T. y López E.A. (2016). Eco-friendly innovation for nejayote coagulation-flocculation process using chitosan: evaluation through zeta potential measurements. Chem. Eng. J. 284 (15), 536-542.

DOI: 10.1016/j.cej.2015.09.026

Tejada M. y González J.L. (2005). Beet vinasse applied to wheat under dryland conditions affects soil properties and yield. Eur. J. Agron. 23 (4), 336-347.

DOI: 10.1016/j.eja.2005.02.005

Téllez V., López J.F., Aragón A. y Zayas T. (2016). Application of nejayote as a foliar and edaphic fertiliser to native blue maize (Zea mays L.) crops. American Journal of Plant Sciences 7, 2221-2238.

DOI: $10.4236 /$ ajps.2016.715196
Uicab-Brito L.A. y Sandoval-Castro C.A. (2003). Uso del contenido ruminal y algunos residuos de la industria cárnica en la elaboración de composta. Trop. Subtrop. Agroecosyst. 2 (2), 45-63.

Valderrama-Bravo C., Gutiérrez-Cortez E., ContrerasPadilla M., Rojas-Molina I., Mosquera J. C., RojasMolina A., Beristáin F. y Rodríguez-García M.E. (2012). Constant pressure filtration of lime water (nejayote) used to cook kernels in maize processing. J. Food Eng. 110 (3), 478-486. DOI:10.1016/j.jfoodeng.2011.12.018

Watson A.S. y Ramstad E.D. (1987). Structure and composition. En: Corn: chemistry and technology (White P.J. y Johnson L.A., Eds.). 2a ed. American Association of Cereal Chemists, Saint Paul, Minnesota, EUA, pp. 69-106.

Williams J.F. (2010). Rice nutrient management in California. UCANR Publications, Oakland, California, EUA, 136 pp.

Yerima B.P.K., Tiamgne Y.A., Fokou L., Tziemi T.C.M.A. y Van Ranst E. (2015). Effect of substrates on germination and seedling emergence of sunflower (Helianthus annuus L.) at the Yongka Western Highlands Research/ Garden Park, BamendaCameroon. Tropicultura 33 (2), 91100.

Zárate B.H. (2013). Valorización de subproductos agrícolas y forestales como sustratos de cultivo en e estado de Oaxaca (México). Tesis de Doctorado. Escuela Técnica Superior de Ingenieros Agrónomos, Universidad Politécnica de Madrid, España, 405 pp. 